\title{
2 Von der Dekonstruktion von Geschäftsfeldern bis zur Disruption von Geschäftsmodellen
}

\author{
Pierre-Michael Meier
}

Seit nunmehr elf Jahren engagiere ich mich über den EVKM, Europäischer Verband der Krankenhaus Manager, ehrenamtlich im Steuerungsgremium der ENTSCHEIDERFABRIK. In diesem sicher ältesten Inkubator für Digitalisierungsprojekte in der Gesundheitsbranche wurden mittlerweile weit mehr als 150 Projekte der Informations-, Kommunikations-, Leit- und Medizintechnik umgesetzt. So konnte schon oft gezeigt werden, welchen nutzenstiftenden Beitrag Digitalisierungsprojekte zum Unternehmenserfolg leisten.

Die Wortwahl nutzenstiftend ist eine positive Kommunikation. Als Bürger dieses Landes kann man zu der Wahrnehmung gelangen, dass Digitalisierung durchweg positiv betrachtet wird. Etwa wenn man sich vor Augen führt, dass laut Statistikportal statista 49 Millionen Deutsche über ein Smartphone verfügen.

Schaut man sich die Wortwahl Dekonstruktion von Geschäftsprozessen bis zu Disruption von Geschäftsmodellen an, so ist der Eindruck richtig, dass hier eine Risikokommunikation gewählt wurde. Was wird die Folge der Digitalisierung sein, wenn die Akteure die Veränderungen nicht antizipieren geschweige denn mitgestalten?

Erfahrungen in der digitalen Disruption machen wir täglich als „Konsument“. Mitglieder der ENTSCHEIDERFABRIK erlebten die Disruption in der Gesundheitswirtschaft 2016 sehr eindringlich auf der Entscheider-Reise in die USA zum Leadership Summit der American Hospital Association in San Diego. Die Frage, die überall gestellt wird, ist, wie der Patient und sein Smartphone bzw. der Bürger mit seiner individuellen Gesundheitsakte oder persönlichen Konsumenten-Patientenakte im Zusammenwirken mit den institutionellen Patientenakten der Leistungserbringer die Prozesse und Strukturen verändern wird, und zwar ohne Rücksicht auf die Normen 
und Regeln unserer Selbstverwaltung. In San Diego wurde den Mitreisenden Antworten gegeben und sie konnten sich davon überzeugen, wie Klinikgruppen bzw. ganze Healthcare-Systeme bestehend aus Leistungserbringern und Kostenträgern mit bis zu 94 Anbietern von individuellen Gesundheitsakten in sämtlichen prä-akutstationären, akutstationären und post-akutstationären Prozessschritten interagierten. Patienten griffen je nach Bedürfnis oder Indikation mit unterschiedlichsten mobilen Smartphone-Apps auf eben diese individuellen Gesundheitsakten zu und Daten wurden mit den institutionellen Patientenakten, z.B. der Kliniken, mehrmals im Behandlungsprozess ausgetauscht.

Zurückkommend auf den oben eingeführten Terminus Risikokommunikation sollten die Betreiber von institutionellen Patientenakten, d.h. Leistungserbringer und Kostenträger, hinterfragen, ob sie zu dieser Interaktion mit individuellen Gesundheitsakten schon fähig sind. Ganz konkret ist zu fragen, ob ein Strategic Health Information Management (HIM) und operativ technisch, eine IHE-konforme Plattformstrategie vorliegen, um die Effizienzpotenziale in der Prozessorganisation tatsächlich nutzen zu können, die durch die Interaktion zwischen dem Patienten bzw. Datenkonsumenten und seiner persönlichen Gesundheitsakte und der institutionellen Patientenakte freigesetzt werden. Auf Neudeutsch hieße das Digitalisierung 4.o.

Das HIM ist in Zeiten knapper Budgets mehr denn je die Lösung des Problems. Strategisches HIM ist der Schlüssel zur Erlössicherung. Auf der einen Seite liefert ein strategisches HIM einen nutzenstiftenden Beitrag zum Unternehmenserfolg bzw. einen Wettbewerbsvorteil gegenüber allen Marktbegleitern, die die Forderungen des Patienten nach einer Interaktion zwischen seiner Gesundheitsakte und den institutionellen Patientenakten nicht erfüllen können und sei es lediglich zu den in Bälde startenden Buchungsportalen à la „Booking.com“.

Auf der anderen Seite ist die IT selbst ein kritischer Unternehmensprozess bzw. maßgebliches Steuerungsinstrument für die Betriebsführung, das nach den Best Practices des IT-Sicherheitsgesetzes als kritische Infrastruktur einzuschätzen und zu schützen ist.

In der Konsequenz heißt das, dass der traditionelle Dreiklang für den Erfolg von Kliniken, d.h. Überschüsse, Auslastung und Investitionen, sich nicht mehr durch eine qualitative und wirtschaftliche Leistungserbringung ergeben wird bzw. letzteres, im Mittelpunkt stehend, den Dreiklang unterstützen bzw. bedingen kann.

Gehen wir auf die Risikokommunikation bzw. die Wortwahl Dekonstruktion von Geschäftsprozessen bis zu Disruption von Geschäftsmodellen näher ein, so sollte dem laut dem statistischem Jahrbuch existenten Durchschnittsbürger klar geworden sein, dass nur die Organisationen im Wettbewerb bestehen werden, die die digitalen Veränderungen antizipieren und annehmen werden.

Beispiele aus anderen Branchen liegen mittlerweile in reichlicher Anzahl vor. Große Umwälzungen fanden in der Reisebranche, im Zeitungsgeschäft, im Einzelhandel etc. statt. Was macht also ein Krankenhaus so viel anders als die Deutsche Bahn oder eine Fluglinie?

Es sind die Menschen, die als Passagiere per App „einchecken“ oder sich „onboarden“, d.h. zu einem wesentlichen Teil des Information Managements dieser Unternehmen werden und Tätigkeiten übernehmen, die vorher „mit der Hand am Arm“ von Mitarbeitern eingegeben werden mussten. Wer kann sich noch daran erinnern, wie 


\section{Qualität}

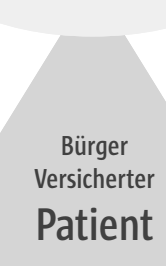

Health Information

Exchange (HIE)

Pay for...

- Quality

- Performance

- HIE

Abb. 1 Der neue „Dreiklang-HIM“ muss auf Qualität, Health Information Exchange (HIE) und aus der Digitalisierung abzuleitenden Finanzierungsmodellen wie „Pay for Quality,

Performance etc." fußen.

diese Unternehmen ohne die maßgebliche Mitwirkung der Passagiere und der Digitalisierung mit ihren Konsumenten interagiert oder Informationen ausgetauscht bzw. zusammengearbeitet haben?

Kennen Ihre Kinder noch die Zeit des Papiertickets?

Der neue Dreiklang, also HIM - Strategic Heath Information Management (s. Abb. 1) muss somit auf Qualität, Health Information Exchange (HIE) und aus der Digitalisierung abzuleitenden Finanzierungsmodellen à la „Pay for Quality, Performance, etc." fußen und stellt somit den Konsumenten, d.h. den Bürger, Versicherten und Patienten als Treiber der Digitalisierung in den Mittelpunkt. Der Konsument wird den Erfolg bestimmen. 